\title{
Computing Subgraph Probability of Random Geometric Graphs: Quantitative Analyses of Wireless Ad Hoc Networks
}

\author{
Chang Wu Yu and Li-Hsing Yen \\ Department of Computer Science and Information Engineering, \\ Chung Hua University, Taiwan, R. O. C \\ \{cwyu, lhyen\}@ chu.edu.tw
}

\begin{abstract}
This paper undergoes quantitative analyses on fundamental properties of ad hoc networks including estimating the number of hiddenterminal pairs and the number of exposed-terminal sets. To obtain these results, we propose a paradigm to systematically derive exact formulas for a great deal of subgraph probabilities of random geometric graphs. In contrast to previous work, which established asymptotic bounds or approximation, we obtain closed-form formulas that are fairly accurate and of practical value.
\end{abstract}

Keywords: Ad hoc networks, sensor networks, analytical method, random geometric graphs, performance evaluation, hidden terminal, exposed terminal, quantitative analysis.

\section{Introduction}

Ad hoc networks (MANETs), which are wireless networks with no fixed infrastructure, have received extensive attentions [1, 5, 8, 12, 38-41, 46, 49-52]. Each mobile node in the network functions as a router that discovers and maintains routes for other nodes. These nodes may move arbitrarily, and therefore network topology changes frequently and unpredictably. Other limitations of ad hoc networks include high power consumption, scare bandwidth, and high error rates. Applications of ad hoc networks are emergency search-and-rescue operations, meetings or conventions in which persons wish to quickly share information, data acquisition operations in inhospitable terrain, and automated battlefield [38]. Bluetooth networks [53] and sensor networks [35, 42] are commercial products of ad hoc networks.

A geometric graph $G=(V, r)$ consists of nodes placed in 2-dimension space $R^{2}$ and edge set $E=\{(i, j) \mid d(i, j) \leq r$, where $i, j \in V$ and $d(i, j)$ denotes the Euclidian distance between node $i$ and node $j\}$. Let $X_{n}=\left\{x_{1}, x_{2}, \ldots, x_{n}\right\}$ be a set of independently and uniformly distributed random points. We use $\Psi\left(X_{n}, r, A\right)$ to denote the random geometric graph (RGG) [29] of $n$ nodes on $X_{n}$ with radius $r$ and placed in an area $A$. RGGs consider geometric graphs on random point configurations. Applications of RGGs include communications networks, classification, spatial statistics, epidemiology, astrophysics, and neural networks [29].

A RGG $\Psi\left(X_{n}, r, A\right)$ is suitable to model an ad hoc network $N=(n, r, A)$ consisting of $n$ mobile devices with transmission radius $r$ unit length that are independently and uniformly 
distributed at random in an area $A$. When each vertex in $\Psi\left(X_{n}, r, A\right)$ represents a mobile device, each edge connecting two vertices represents a possible communication link as they are within the transmission range of each other. A random geometric graph and its representing network are shown in Figure 1. In the example, area $A$ is a rectangle that is used to model the deployed area such as a meeting room. Area $A$, however, can be a circle, or any other shape, and even infinite space.

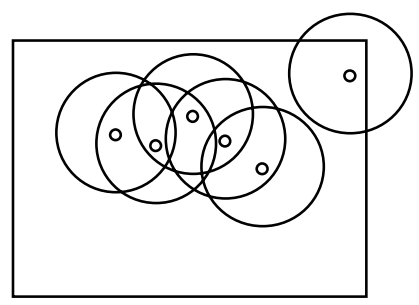

(a)

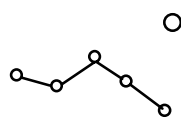

(b)

Fig. 1. (a) An ad hoc network $N=(6, r, A)$, where $A$ is a rectangle. (b) Its associated random geometric graph $\Psi\left(X_{6}, r, A\right)$.

RGGs are different from well-known random graphs $[3,13,28]$. One kind of random graph can be characterized by two parameters $n$ and $p$, where $n$ represents the number of nodes and $p$ represents the probability of the existence of each possible edge. Edge occurrences in the random graph are independent to each other, which is not the case in MANETs. Therefore the fruitful results of random graphs cannot be directly applied to MANETs. Other graph models proposed for MANETs are interval graphs [16], unit disk graph [7, 17], proximity graphs [29], and indifference graphs [37].

Many fundamental properties of ad hoc networks are related to subgraphs in RGGs. For example, the IEEE 802.11 CSMA/CA protocol suffers from the hidden and the exposed terminal problem [41, 45]. The hidden terminal problem is caused by concurrent transmissions of two nodes that cannot sense each other but transmit to the same destination. We call such two terminals a hidden-terminal pair. The existence of hidden-terminal pairs in an environment seriously results in garbled messages and increases communication delay, thus degrading system performance $[24,25,45]$.

A hidden-terminal pair can be represented by a pair of edges $(x, y)$ and $(x, z)$ of $G=(V, E)$ such that $(x, y) \in E$ and $(x, z) \in E$, but $(y, z) \notin E$. In graph terms, such a pair of edges is an induced subgraph $p_{2}$ that is a path of length two (See Figure 2). Counting the occurrences of $p_{2}$ in a given RGG helps counting the number of hidden-terminal pairs in the network.

The exposed terminal problem is due to prohibiting concurrent transmissions of two nodes that sense each other but can transmit to different receivers without conflicts [41]. The problem results in unnecessary reduction in channel utilization and throughput. We name these nodes an exposed-terminal set. Similarly, the problem can be modeled as a subgraph $H$ of $G=(V, E)$ with four vertices $\{x, y, z, w\} \subseteq V$ such that $\{(x, y),(y, z),(z, w)\} \subseteq E$, but $(x, z) \notin E$ and $(y, w) \notin E$ (See Figure 2). 

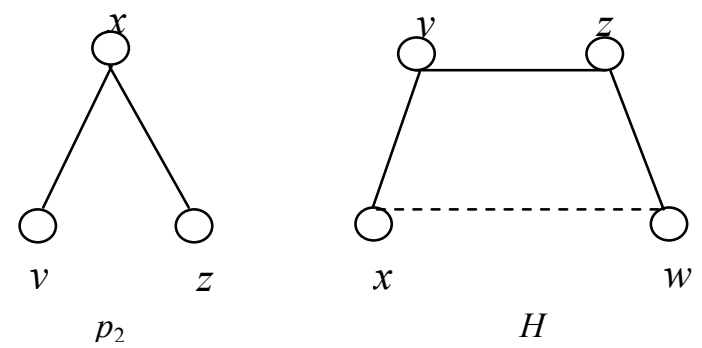

Fig. 2. The subgraphs of hidden-terminal pair $p_{2}$ and exposed-terminal set $H$

Quantitative analyses on specific subgraphs of a given RGG are of importance for understanding and evaluating the fundamental properties of MANETs. There is extensive literature on the subgraph probability of RGGs [29]. Penrose had shown that, for arbitrary feasible connected subgraph $\Gamma$ with $k$ vertices, the number of induced subgraphs isomorphic to $\Gamma$ satisfies a Poisson limit theorem and a normal limit theorem [29]. To the best of our knowledge, previous related results are all asymptotic or approximate.

In the paper, we make the first attempt to propose a paradigm to systematically derive the exact formulas for a great deal of subgraph probabilities in RGGs. In contrast to previous asymptotic bounds or approximation, the closed-form formulas we derived are fairly accurate and of practical value. With the paradigm, we undergoes quantitative analyses on fundamental properties of ad hoc networks including the number of hidden-terminal pairs and the number of exposed-terminal sets.

Computing the probability of occurrence of RGG subgraphs is complicated by the assumption of finite plane. For example, one device in Figure 1 is deployed nearby the boundary of rectangle $A$ so its radio coverage region (often modeled by a circle) is not properly contained in $A$. This is due to border effects, which complicate the derivation of closed formulas. Previous discussions usually circumvent the border effects by using torus convection $[1,20]$. Torus convention models the network topology in a way that nodes nearby the border are considered as being close to nodes at the opposite border and they are allowed to establish links. Most of the time, we adopt torus convention to deal with border effects in the paper. However, we also obtain an exact formula for the single edge probability of RGGs when confronting the border effects.

Our definition of random geometric graphs $\Psi\left(X_{n}, r, A\right)$ is different from those of Poisson point process $[1,12]$, which assume that the distribution of $n$ points (vertices) on a possibly infinite plane follows a Poisson distribution with parameter $\lambda$ (the given density). In Poisson point process, the number of vertices can only be a random number rather than a tunable parameter. In practice, however, some MANET modeling requires a fixed input $n$ or a finite deployed area.

The rest of the paper is organized as follows. In Section 2, some definitions and notations are introduced. In Section 3, we briefly survey related results on RGGs. A paradigm for computing the subgraph probability of RGGs with torus convention is presented in Section 4. Section 5 presents those derivations when confronting border effects. In Section 6, quantitative analyses on ad hoc networks are discussed. Finally, Section 7 concludes the paper. 


\section{Definitions and Notations}

A graph $G=(V, E)$ consists of a finite nonempty vertex set $V$ and edge set $E$ of unordered pairs of distinct vertices of $V$. A graph $\mathrm{G}=(V, E)$ is labeled when the $|V|$ vertices are distinguished from one another by names such as $v_{1}, v_{2}, \ldots, v_{\mid \eta}$. Two labeled graphs $G=\left(V_{G}\right.$, $\left.E_{G}\right)$ and $H=\left(V_{H}, E_{H}\right)$ are identical, denoted by $G=H$ if $V_{G}=V_{H}$ and $E_{G}=E_{H}$. A graph $H=\left(V_{H}\right.$, $\left.E_{H}\right)$ is a subgraph of $G=\left(V_{G}, E_{G}\right)$ if $V_{H} \subseteq V_{G}$ and $E_{H} \subseteq E_{G}$. Suppose that $V^{\prime}$ is a nonempty subset of $V$. The subgraph of $G=(V, E)$ whose vertex set is $V^{\prime}$ and whose edge set is the set of those edges of $G$ that have both ends in $V^{\prime}$ is called the subgraph of $G$ induced by $V^{\prime}$, denoted by $G_{V}$. The size of any set $S$ is denoted by $|S|$. The degree of a vertex $v$ in graph $G$ is the number of edge incident with $v$. The notation $\left(\begin{array}{l}n \\ m\end{array}\right)$ denotes the number of ways to select $m$ from $n$ distinct objects.

The subgraph probability of RGGs is defined as follows. Let $\Omega=\left\{G_{1}, G_{2}, \ldots, G_{k}\right\}$ represent every possible labeled graphs of $\Psi\left(X_{n}, r, A\right)$, where $k=2$. When $G_{x}$ is a labeled subgraph in $\Omega$, we use $\operatorname{Pr}\left(G_{x}\right)$ to denote the probability of the occurrence of $G_{x}$. Suppose $S \subseteq V$ and $T \subseteq V$, we define $\operatorname{Pr}\left(G_{s}\right)=\sum_{\forall G_{w} \in \Omega \text { and } G_{s} \subseteq G_{w}} \operatorname{Pr}\left(G_{w}\right)$, when $1 \leq w \leq k$.

A walk in $G=(V, E)$ is a finite non-null sequence $W=v_{0} e_{1} v_{1} e_{2} \ldots e_{k} v_{k}$, where $v_{i} \in V$ and $e_{j} \in E$ for $0 \leq i \leq k$ and $1 \leq j \leq k$. The integer $k$ is the length of the walk. When $v_{0}, v_{1}, \ldots, v_{k}$ are distinct, $W$ is called a path. A path is a cycle if its origin and terminus are the same. An induced subgraph that is a path of length $i$ is denoted by $p_{i}$. Similarly, an induced subgraph that is a cycle of length $i$ is denoted by $c_{i} ; c_{3}$ is often called a triangle. A set of vertices is independent if no two of them are adjacent. An induced subgraph which is an independent set of size $i$ is denoted by $I_{i}$. The notational conventions used in the paper can be found in [4].

\section{Related Work in RGG}

A book written by Penrose [29] provides and explains the theory of random geometric graphs. Graph problems considered in the book include subgraph and component counts, vertex degrees, cliques and colorings, minimum degree, the largest component, partitioning problems, and connectivity and the number of components.

For $n$ points uniformly randomly distributed on a unit cube in $d \geq 2$ dimensions, Penrose [32] showed that the resulting geometric random graph $G$ is $k$-connected and $G$ has minimum degree $k$ at the same time when $n \rightarrow \infty$. In [9, 10], Díaz et al. discussed many layout problems including minimum linear arrangement, cutwidth, sum cut, vertex separation, edge bisection, and vertex bisection in random geometric graphs. In [11], Díaz et al. considered the clique or chromatic number of random geometric graphs and their connectivity.

Some results of RGGs can be applied to the connectivity problem of ad hoc networks. In [39], Santi and Blough discussed the connectivity problem of random geometric graphs $\Psi\left(X_{n}, r, A\right)$, where $A$ is a $d$-dimensional region with the same length size. In [1], Bettstetter investigated two fundamental characteristics of wireless networks: its minimum node degree 
and its $k$-connectivity. In [12], Dousse et al. obtained analytical expressions of the probability of connectivity in the one dimension case. In [18], Gupta and Kumar have shown that if $r=\sqrt{\frac{\log n+c(n)}{\pi n}}$, then the resulting network is connected with high probability if and only if $c(n) \rightarrow \infty$. In [47], Xue and Kumar have shown that each node should be connected to $\Theta(\log$ $n$ ) nearest neighbors in order that the overall network is connected.

Recently, Yen and Yu have analyzed link probability, expected node degree, and expected coverage of MANETs [49]. In [48], Yang has obtained the limits of the number of subgraphs of a specified type which appear in a random graph.

\section{A Paradigm for Computing Subgraph Probability}

In the section, we develop a paradigm for computing subgraph probability of RGGS. First of all, we are to prove that the occurrences of arbitrary two distinct edges in RGGs are independent in the next subsection. The property of edge independence greatly simplifies our further calculations. For simplicity, we always assume that $A$ is sufficiently large to properly contain a circle with radius $r$ in a $\Psi\left(X_{n}, r, A\right)$ throughout the paper; that implies $\pi r^{2} \leq|A|$. In the paper, notation $E_{i}\left(E_{i}^{\prime}\right)$ denotes the event of the occurrence (absence) of edge $e_{i}$.

Since we adopt torus convention to avoid border effects in the section, single-edge probability in RGG is obtained trivially and listed below.

Theorem 1: We have $\operatorname{Pr}\left(E_{j}\right)=\pi r^{2}|A|$, for an arbitrary edge $e_{j}=(u, v)$ and $u \neq v$, in a $\Psi\left(X_{n}, r, A\right)$.

\subsection{Edge Independence in RGGs}

The next theorem will indicate that the occurrences of arbitrary two distinct edges in RGGs are independent. The result is somewhat difficult to be accepted as facts at first glance for some scholars. The following theorem shows that the occurrences of arbitrary two distinct edges in RGGs are independent even if they share one end vertex.

Theorem 2 [49]: For arbitrary two distinct edges $e_{i}=(u, v)$ and $e_{j}=(w, x)$ in a $\Psi\left(X_{n}, r, A\right)$, we have $\operatorname{Pr}\left(E_{i} E_{j}\right)=\operatorname{Pr}\left(E_{i}\right) \operatorname{Pr}\left(E_{j}\right)$.

Note that Theorem 2 does not imply that the occurrences of more than two edges in RGGs are also independent. In fact, we will show their dependence later.

By Theorem 1 and 2, we obtain the probability of two-edge subgraphs immediately.

Corollary 3: For arbitrary two distinct edges $e_{i}=(u, v)$ and $e_{j}=(w, x)$ in a $\Psi\left(X_{n}, r, A\right)$, we have $\operatorname{Pr}\left(E_{i} E_{j}\right)=\left(\pi r^{2} /|A|\right)^{2}$.

\subsection{Base Subgraphs}

In this subsection, we consider eight labeled subgraphs with three vertices as base subgraphs, the probabilities of which will be used to compute the probability of larger subgraphs later. Based on the number of edges included, subgraphs of three vertices can be classified into four groups: a triangle $\left(c_{3}\right)$, an induced path of length two $\left(p_{2}\right)$, an edge with an isolated vertex $\left(p_{1}+I_{1}\right)$, and three isolated vertices $\left(I_{3}\right)$ (See Figure 3 ).

To compute the probability of $c_{3}$, we need the following lemma. Two equal-sized circles are properly intersecting if one circle contains the center of the other. Due to page limit, we omit the proofs of Lemma 4-5 and Theorem 6- 9 intentionally. 


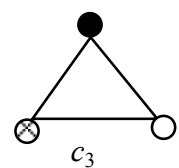

$c_{3}$

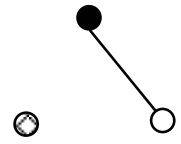

$p_{1}+I_{1}$
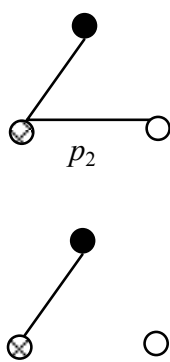

$p_{1}+I_{1}$

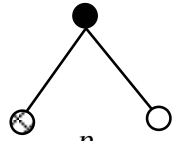

$p_{2}$

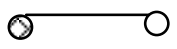

$p_{1}+I_{1}$

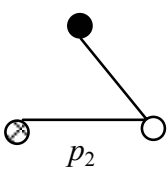

0

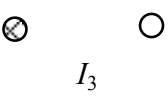

Fig. 3. Eight base subgraphs

Lemma 4: The expected overlapped area of two properly intersecting circles with the same radius $r$ is $\left(\pi-\frac{3 \sqrt{3}}{4}\right) r^{2}$ in a $\Psi\left(X_{n}, r, A\right)$.

The following conditional probability is a consequence of Lemma 4.

Lemma 5: For three distinct edges $e_{i}=(u, v), e_{j}=(u, w)$, and $e_{k}=(v, w)$ in a $\Psi\left(X_{n}, r, A\right)$, we have $\operatorname{Pr}\left(E_{i} E_{j} \mid E_{k}\right)=\left(\pi-\frac{3 \sqrt{3}}{4}\right) r^{2} /|A|$, where $u \neq v \neq w$.

The probability of the first base subgraph $c_{3}$ (triangle) can then be obtained.

Theorem 6: For three distinct edges $e_{i}=(u, v), e_{j}=(u, w)$, and $e_{k}=(v, w)$ in a $\Psi\left(X_{n}, r, A\right)$, we have $\operatorname{Pr}\left(E_{i} E_{j} E_{k}\right)=\left(\pi-\frac{3 \sqrt{3}}{4}\right) \pi r^{4} /|A|^{2}$, where $u \neq v \neq w$.

Next, we consider the subgraph of an edge with an isolated vertex $\left(p_{1}+I_{1}\right)$.

Theorem 7: For three distinct edges $e_{i}=(u, v), e_{j}=(u, w)$, and $e_{k}=(v, w)$ in a $\Psi\left(X_{n}, r, A\right)$, we have $\mathrm{P}\left(E_{i} E_{j}^{\prime} E_{k}^{\prime}\right)=\frac{\pi r^{2}}{|A|}\left(1-\frac{\pi r^{2}}{|A|}-\frac{3 \sqrt{3}}{4|A|} r^{2}\right)$, where $u \neq v \neq w$.

We have shown that the occurrences of two distinct edges in a $\Psi\left(X_{n}, r, A\right)$ are independent (Theorem 2). The next theorem, however, shows that edge independence does not exist for subgraphs with three or more edges.

Theorem 8: The occurrences of arbitrary three distinct edges in a $\Psi\left(X_{n}, r, A\right)$ are dependent.

The next base subgraph we considered is an induced path $p_{2}$, which will be used to model a hidden-terminal pair.

Theorem 9: For arbitrary three distinct edges $e_{i}=(u, v), e_{j}=(u, w)$, and $e_{k}=(v, w)$ in a $\Psi\left(X_{n}, r\right.$, $A$ ), we have $\operatorname{Pr}\left(E_{i} E_{j} E_{k}{ }^{\prime}\right)=\left(\frac{3 \sqrt{3}}{4}\right) \pi r^{4} /|A|^{2}$, where $u \neq v \neq w$.

The last base subgraph we considered is $I_{3}$.

Theorem 10: For arbitrary three distinct edges $e_{l}=(u, v), e_{j}=(u, w)$, and $e_{k}=(v, w)$ in a $\Psi\left(X_{n}, r\right.$, $A$ ), we have $\operatorname{Pr}\left(E_{i}^{\prime} E_{j}^{\prime} E_{k}^{\prime}\right)={ }_{1}-\frac{\pi r^{4}}{|A|}-\frac{\frac{3 \sqrt{3}}{4}}{|A|^{2}} \pi r^{4}$, where $u \neq v \neq w$.

Proof: (Omitted.) 


\subsection{A Paradigm for Computing Subgraph Probability of RGGs}

To simplify calculation, we adopt the following graph drawings. A solid line denotes an edge of $G$; a broken line denotes a possible edge between them; two vertices without a line denote a non-edge of $G$. Note that such graph drawing represent a class of graphs $G=\left(V, E_{S}\right.$, $\left.E_{B}\right)$, where $E_{S}\left(E_{B}\right)$ denotes solid-line edge (broken-line edge) set. For example, the following graph denotes eight base graphs depicted in Figure 3.

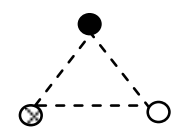

We list some subgraphs discussed in Section 4.1 or 4.2 with their notations, drawings, and probabilities in Table 1 .

Table 1. Probabilities of subgraphs with three vertices or less in a RGG

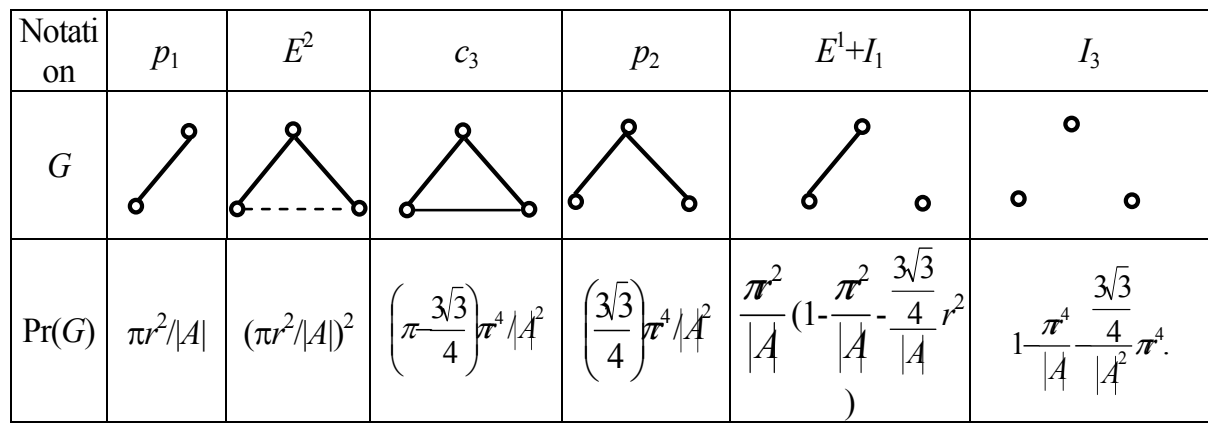

Note that we have $\operatorname{Pr}\left(E^{2}\right)=\operatorname{Pr}\left(c_{3}\right)+\operatorname{Pr}\left(p_{2}\right)$ in Table 1 . This equation can be derived by the following two types of derivation rules.

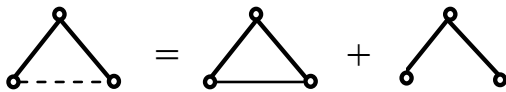

Type

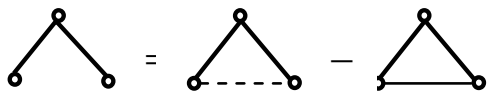

Type II

In fact, type I (type II) graph derivation rule can be applied on any broken-line edge (non-edge) of any graph. That is, for any $e \in E_{B}$, we have $G\left(V, E_{S}, E_{B}\right)=G_{1}\left(V, E_{S} \cup\{e\}\right.$, $\left.E_{B^{-}}\{e\}\right)+G_{2}\left(V, E_{S}, E_{B^{-}}\{e\}\right)$. Similarly, for any $e \notin E_{S} \cup E_{B}$, we have $G\left(V, E_{S}, E_{B}\right)=G_{1}\left(V, E_{S}\right.$, $\left.E_{B} \cup\{e\}\right)-G_{2}\left(V, E_{S} \cup\{e\}, E_{B}\right)$ equivalently. We will show how these derivation rules can be used to systematically compute subgraph probability of RGGs.

Given a subgraph of a RGG, we try to obtain its probability by following three basic steps in the paradigm:

(1) Decompose the graph into a linear combination of base graphs by recursively applying the derivation rules.

(2) Compute the probabilities of base graphs. 
(3) Compute the probability of the graph by manipulating the probabilities of base graphs.

We have established probability formulas for essential components (i.e. base graphs) in Section 4.2. The following example demonstrates the great convenience of this paradigm. A graph $H$ (representing the exposed-terminal set) is decomposed into a set of subgraphs according to the derivation rules.

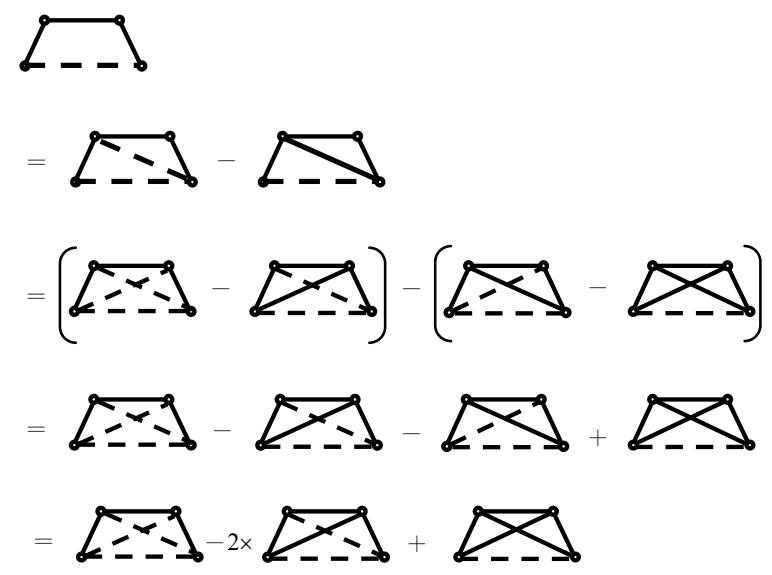

Graph $H$ turns out to be a linear combination of three graphs. Although these subgraphs are not base graphs, we can obtain their probabilities with the help of base graphs. The first graph (denoted by $E^{3}$ ) consists of three solid edges (which form a path of length three) and three other broken edges; therefore we can obtain its probability by applying Theorem 1 three times; that is, we have $\operatorname{Pr}\left(E^{3}\right)=\left(\pi r^{2} /|A|\right)^{3}$. The second graph (denoted by $\left.E^{1}+c_{3}\right)$ consists of a triangle and a solid edge; then its probability can be obtained by applying Theorem 6 and Theorem 1 once; that is, we have $\operatorname{Pr}\left(E^{1}+c_{3}\right)=\left(\pi-\frac{3 \sqrt{3}}{4}\right) \frac{\pi r^{4}}{|A|^{2}} \times \frac{\pi r^{2}}{|A|}$. The last graph (denoted by $c_{3}{ }^{2}$ ) consists of two triangles with a common edge; we can also obtain its probability by applying Theorem 1 once and Lemma 5 twice; that is, we have $\operatorname{Pr}\left(c_{3}{ }^{2}\right)=\left(\left(\pi-\frac{3 \sqrt{3}}{4}\right) \frac{r^{2}}{|A|}\right)^{2} \times \frac{\pi r^{2}}{|A|}$. According to above discussion, we have $\operatorname{Pr}(H)=\operatorname{Pr}\left(E^{3}\right)-2 \times \operatorname{Pr}\left(E^{1}+c_{3}\right)+\operatorname{Pr}\left(c_{3}^{2}\right)=\left(\pi r^{2} / A\right)^{3}-2 \times\left(\pi-\frac{3 \sqrt{3}}{4}\right) \frac{\pi^{2} r^{6}}{|A|^{3}}+\left(\pi-\frac{3 \sqrt{3}}{4}\right)^{2} \frac{\pi r^{6}}{|A|^{3}}=\frac{27}{16} \frac{\pi r^{6}}{|A|^{3}}$.

In summary, we have the following theorem.

Theorem 11: For arbitrary four distinct nodes $x, y, z$, and $w$ in a $\Psi\left(X_{n}, r, A\right)$, we have $\operatorname{Pr}\left(G_{S}=H\right)=\frac{27}{16} \frac{\pi r^{6}}{|A|^{3}}$, where $S=\{x, y, z, w\}$ and $H=\left(V_{H}, E_{H}\right)$ with $V_{H}=S$ and $\{(x, y),(y, z),(z$, $w)\} \subseteq E_{H}$, but $(x, z) \notin E_{H}$ and $(y, w) \notin E_{H}$.

Table 2 lists subgraphs and associated probabilities mentioned above. 
Table 2. Probabilities of some subgraphs with four vertices in a RGG

\begin{tabular}{|c|c|c|c|c|}
\hline Notation & $E^{3}$ & $E^{1}+c_{3}$ & $c_{3}{ }^{2}$ & $H$ \\
\hline G & & & & \\
\hline $\operatorname{Pr}(G)$ & $\left(\pi r^{2} /|A|\right)^{3}$ & $\left(\pi-\frac{3 \sqrt{3}}{4}\right) \frac{\pi^{2} r^{6}}{|A|^{3}}$ & $\left(\pi-\frac{3 \sqrt{3}}{4}\right)^{2} \frac{\pi r^{6}}{|A|^{3}}$ & $\frac{27}{16} \frac{\pi r^{6}}{|A|^{3}}$ \\
\hline
\end{tabular}

Following our paradigm, the probability formulas of a great deal of subgraphs (in RGGs) can be obtained systematically. In Section 6, we will demonstrate that such specific subgraphs with their properties have considerable merit in quantitative analyses of wireless ad hoc networks.

\section{Computing Subgraph Probability in the Face of Border Effects}

In the section, we restrict the deployed area $A$ to an $l \times m$ rectangle. We make an attempt to face border effects and obtain a closed-form formula of computing the single edge probability of RGGs. The results derived in the section can be used to measure the extent of coverage and connectivity of ad hoc networks [23].

Due to page limit, the main result and its corollaries are listed only.

Theorem 12 [49]: Given a $\Psi\left(X_{n}, r, A\right)$ and an $l \times m$ rectangle $A$, the single edge probability considering border effects is $\frac{\frac{1}{2} r^{4}-\frac{4}{3} l r^{3}-\frac{4}{3} m r^{3}+\pi r^{2} m l}{m^{2} l^{2}}$.

Corollary 13: The average (expected) degree of a vertex in a $\Psi\left(X_{n}, r, A\right)$ considering border effects is $(n-1) \times\left(\frac{\frac{1}{2} r^{4}-\frac{4}{3} l r^{3}-\frac{4}{3} m r^{3}+\pi r^{2} m l}{m^{2} l^{2}}\right)$, where $A$ is an $l \times m$ rectangle.

Corollary 14: The expected edge number of a $\Psi\left(X_{n}, r, A\right)$ considering border effects is $\left(\frac{n(n-1)}{2}\right) \times\left(\frac{\frac{1}{2} r^{4}-\frac{4}{3} l r^{3}-\frac{4}{3} m r^{3}+\pi r^{2} m l}{m^{2} l^{2}}\right)$, where $A$ is an $l \times m$ rectangle.

To obtain these results, we first derive some necessary lemmas. Let $X_{n}=\left\{x_{1}, x_{2}, \ldots, x_{n}\right\}$ be a set of independently and uniformly distributed random points in a given $\Psi\left(X_{n}, r, A\right)$, where $x_{i}=\left(X_{i}, Y_{i}\right)$ and $0 \leq X_{i} \leq l$ and $0 \leq Y_{i} \leq m$, for $1 \leq i \leq n$. Clearly, $X_{i}^{\prime}$ s (and $Y_{i}^{\prime}$ s) are independent, identically distributed random variables with probability density function (p.d.f.) $f(x)=1 / l$ $(g(y)=1 / m)$ over the range $[0, l]([0, m])$.

Lemma 15 [49]: Given a $\Psi\left(X_{n}, r, A\right)$ and any two distinct nodes $x_{i}=\left(X_{i}, Y_{i}\right)$ and $x_{j}=\left(X_{j}, Y_{j}\right)$, we have $\operatorname{Pr}\left[\left|X_{i}-X_{j}\right| \leq z\right]=\frac{-z^{2}+2 l z}{l^{2}}$ and $\operatorname{Pr}\left[\left|Y_{i}-Y_{j}\right| \leq w\right]=\frac{-w^{2}+2 m w}{m^{2}}$ where $0 \leq z \leq l$ and $0 \leq w \leq m$. 
Lemma 16 [49]: Given a $\Psi\left(X_{n}, r, A\right)$ and any two distinct nodes $x_{i}=\left(X_{i}, Y_{i}\right)$ and $x_{j}=\left(X_{j}, Y_{j}\right)$, we have that: (1) the p.d.f. of $\left(X_{i}-X_{j}\right)^{2}$ is $f(u)=\frac{l u^{-\frac{1}{2}}-1}{l^{2}}$ where $0 \leq u \leq l^{2}$, and (2) the p.d.f. of $\left(Y_{i}-Y_{j}\right)^{2}$ is $g(v)=\frac{m v^{-\frac{1}{2}}-1}{m^{2}}$, where $0 \leq v \leq m^{2}$.

Lemma 17 [43]: $\int u^{-\frac{1}{2}} \sqrt{a^{2}-u} d u=u^{\frac{1}{2}} \sqrt{a^{2}-u}+a^{2} \sin ^{-1} \frac{\sqrt{u}}{a}+c$, where $c$ is a constant.

We conclude that border effect does affect the value of the single edge probability of $\Psi\left(X_{n}, r, A\right)$. If $A$ is an $l \times m$ rectangle, the difference between the single edge probabilities with and without avoiding border effects (by adopting torus convention) is $\frac{\frac{4}{3} m r^{3}+\frac{4}{3} l r^{3}-\frac{1}{2} r^{4}}{m^{2} l^{2}}$.

\section{Quantitative Analyses of Wireless ad Hoc Networks}

In the section, we make use of the derived results to develop quantitative analyses of ad hoc (sensor) networks including the number of hidden-terminal pairs and the number of exposed-terminal sets.

\subsection{The Number of Hidden-Terminal Pairs}

First, we compute the expected number of hidden-terminal pairs in any RGG. The performance of media access control (MAC) scheme is in close relation to the number of hidden-terminal pair of a given wireless network [24, 25, 45]. In literature, a hidden-terminal pair can be modeled by Hearing graph [45]; RTS/CTS mechanism and other methods have been designed for alleviate the hidden terminal problems [2, 14].

Since each hidden-terminal pair consists of three distinct labeled vertices, we set $S$ to be the selected three-vertex set. There are $\left(\begin{array}{l}n \\ 3\end{array}\right)$ different combinations for selecting three from $n$ vertices, and three different settings for labeling one from three as the center of the hidden-terminal pair (i.e. the internal node of the induced path with length 2). Therefore, we have the number of hidden-terminal pairs $\left(\begin{array}{l}n \\ 3\end{array}\right) \times 3 \times \operatorname{Pr}\left(G_{S}=p_{2}\right)=3\left(\begin{array}{l}n \\ 3\end{array}\right)\left(\frac{3 \sqrt{3}}{4}\right) \pi r^{4} /|A|^{2}$ by

Theorem 9.

Theorem 18: The expected number of hidden-terminal pairs in a $\Psi\left(X_{n}, r, A\right)$ is $3\left(\begin{array}{l}n \\ 3\end{array}\right)\left(\frac{3 \sqrt{3}}{4}\right) \pi r^{4} /|A|^{2}$.

Since $3\left(\begin{array}{l}n \\ 3\end{array}\right)\left(\frac{3 \sqrt{3}}{4}\right) \pi r^{4} /|A|^{2}=3 \times\left(\frac{n \times(n-1) \times(n-2)}{1 \times 2 \times 3}\right) \times\left(\frac{3 \sqrt{3}}{4}\right) \pi r^{4} /|A|^{2}$ $=\left(n^{3}-3 n^{2}+2 n\right)\left(\frac{3 \sqrt{3}}{8}\right) \pi r^{4} /|A|^{2}$, we conclude that the hidden terminal pairs grow as like $O\left(n^{3} r^{4}\right)$, where $n$ is the number of mobile nodes and $r$ is the range of power. 
In [24], Khurana et al. have shown that if the number of hidden terminal pairs is small and when collisions are unlikely, the RTS/CTS exchange is a waste of bandwidth. On the other hand, if the number of hidden terminal pairs is large, RTS/CTS mechanism helps avoid collision. Moreover, the optimal value for the RTS_Threshold in IEEE 802.11 [24] depends on the number of hidden terminals.

In [25], Khurana et al. have shown that hidden terminals can have a detrimental effect on the performance (including throughput, packet delay, and blocking probability) of the IEEE 802.11 MAC protocol. Specifically, they have showed that throughput is acceptable when the number of hidden-terminal pairs is less than $10 \%$, beyond which throughput can fall sharply [25]. When determining a network-level simulation of a mobile ad hoc network or designing a wireless network, we can (with Theorem 18) precisely control the quantity of hidden terminal pairs by adjusting the number of mobile nodes or the power range.

\subsection{The Number of Exposed Terminal Sets}

To derive a tight bound of the number of exposed-terminal sets in a given RGG, we need to compute first the subgraph probability of $c_{4}$ (a cycle of length four). The paradigm proposed in Section 5 can be applied to tackle a great deal of subgraphs, but not some types of subgraphs such as cycles. We try to obtain tight bounds for $\operatorname{Pr}\left(c_{4}\right)$ in a different way.

Theorem 19: For arbitrary four distinct nodes $u, v, w$, and $x$ in a $\Psi\left(X_{n}, r, A\right)$, we have $\operatorname{Pr}\left(G_{S}=c_{4}\right) \leq\left(\frac{3 \sqrt{3}}{4}\right) \frac{\pi r^{6}}{|A|^{3}}$, where $S=\{u, v, w, x\}$.

Proof: Consider the geometric graph $c_{4}$ and its circle model (See Figure 4(a) and Figure 4(b)). These four nodes need to be placed properly near to each other in order to form the cycle of length four. Since the longest distance between every two neighboring centers is $r$, the four centers in the circle model must be placed in a convex quadrilateral with the same size length $r$ (See Figure 4(c)).
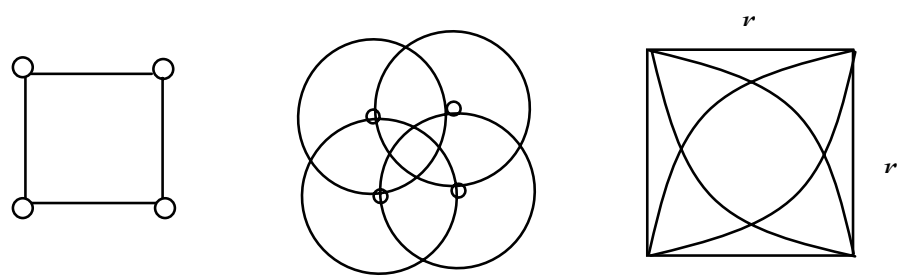

Fig. 4. (a) A cycle of length four. (b) Its circle model. (c) The convex quadrilateral in the circle model.

Since the subgraph $c_{4}$ consists of a induced path $p_{2}$ and another nearby vertex, we have $\operatorname{Pr}\left(G_{S}=c_{4}\right) \leq \operatorname{Pr}\left(G_{S}=p_{2}\right) \times \operatorname{Pr}$ (the remaining vertex is near $p_{2}$ properly). Because $\operatorname{Pr}$ (the remain vertex is near $p_{2}$ properly) is the probability of putting the center of the remaining node in the convex quadrilateral, we have $\operatorname{Pr}\left(\right.$ the remaining vertex is near $p_{2}$ properly) $\leq\left(r^{2} /|A|\right)$. In a sequel, we have $\operatorname{Pr}\left(G_{S}=c_{4}\right) \leq \frac{r^{2}}{|A|} \times\left(\frac{3 \sqrt{3}}{4}\right) \frac{\pi r^{4}}{|A|^{2}}=\left(\frac{3 \sqrt{3}}{4}\right) \frac{\pi r^{6}}{|A|^{3}}$ by Theorem 9. 


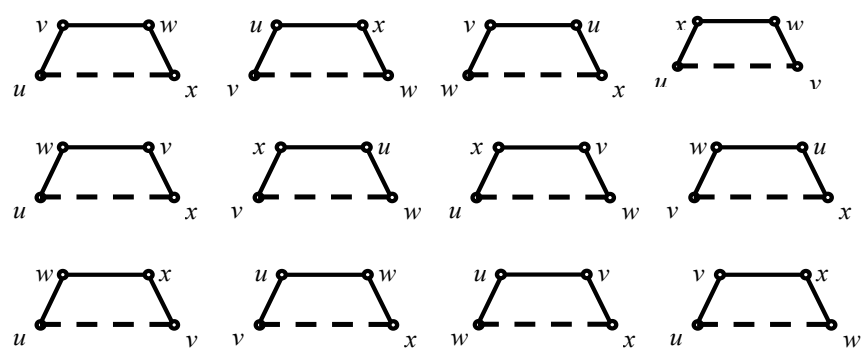

Fig. 5. Twelve different ways of labeling $H$ graph

Counting the number of exposed-terminal sets is equivalent to counting the number of labeled subgraph $H$ (See Table 2). There are $\left(\begin{array}{l}n \\ 4\end{array}\right)$ ways to select four from $n$ elements. Each has $\left(\begin{array}{l}4 \\ 2\end{array}\right) \times 2=12$ ways in forming the subgraph $H$ (Figure 5).

Note that every graph in the same row contains the same subgraph (cycle of length four). Therefore the number of exposed-terminal sets is equal to the number of labeled $H$ graphs minus the number of the duplicated cycles (=3(duplicated counting) $\times 3$ (rows)):

$$
\begin{aligned}
& \left(\begin{array}{l}
4 \\
2
\end{array}\right) \times 2 \times\left(\begin{array}{l}
n \\
4
\end{array}\right) \times \operatorname{Pr}\left(G_{S}=H\right)-3 \times 3 \times\left(\begin{array}{l}
n \\
4
\end{array}\right) \times \operatorname{Pr}\left(G_{S}=c_{4}\right) \\
& =\frac{3^{4}}{4}\left(\begin{array}{l}
n \\
4
\end{array}\right) \frac{\pi r^{6}}{|A|^{3}}-9 \times\left(\begin{array}{l}
n \\
4
\end{array}\right) \times \operatorname{Pr}\left(G_{S}=c_{4}\right) \\
& \geq \frac{3^{4}}{4}\left(\begin{array}{l}
n \\
4
\end{array}\right) \frac{\pi r^{6}}{|A|^{3}}-9 \times\left(\begin{array}{l}
n \\
4
\end{array}\right) \times\left(\frac{3 \sqrt{3}}{4}\right) \frac{\pi r^{6}}{|A|^{3}} \\
& \geq\left(\frac{3^{4}-27 \sqrt{3}}{4}\right)\left(\begin{array}{l}
n \\
4
\end{array}\right) \frac{\pi r^{6}}{|A|^{3}} .
\end{aligned}
$$

Theorem 20: The expected number of exposed-terminal sets in a $\Psi\left(X_{n}, r, A\right)$ is no less than $\left(\frac{3^{4}-27 \sqrt{3}}{4}\right)\left(\begin{array}{l}n \\ 4\end{array}\right) \frac{\pi r^{6}}{|A|^{3}}$.

Similarly, we conclude that the exposed-terminal sets grow as like $O\left(n^{4} r^{6}\right)$, where $n$ is the number of mobile nodes and $r$ is the range of power. In [41], Shukla et al. have mitigated the exposed terminal problem by identifying exposed terminal sets and scheduling concurrent transmissions whenever possible. Combing with Theorem 20, we can estimate the extent of performance degradation due to the exposed-terminal problem, and adopt similar techniques used in [41] to improve system performance.

\section{Conclusions}

We have proposed a paradigm for computing the subgraph probabilities of RGGs, and have shown its applications in finding fundamental properties of wireless networks. We are surprised at finding some interesting properties: 
1. The occurrences of two distinct edges in RGG are independent.

2. The occurrences of three or more distinct edges in RGG are dependent.

3. Probabilities of some specific subgraphs in RGG can be estimated accurately.

Many interesting subgraph probabilities and their applications in MANETs are still uncovered. For example, we are now interested in accurately estimating the diameter of RGGs. We also believe that the techniques developed in the paper can be exploited to conduct quantitative analysis on other fundamental properties of wireless ad hoc networks.

\section{Acknowledgements}

We would like to thank Dr. Jau-Ling Shih for her invaluable help.

\section{References}

1. Christian Bettstetter, "On the minimum node degree and connectivity of a wireless multihop network," MobiHoc, 2002, pp. 80-91.

2. V. Bharghavan, A. Demers, S. Shenker, and L. Zhang, "MACAW: a media access protocol for wireless LANs," ACM SIGCOMM, 1994, pp. 212-215.

3. B. Bollobas, Random Graphs, London: Academic Press, 1985.

4. J. A. Bondy and U. S. R. Murty, Graph Theory with Applications, Macmillan Press, 1976.

5. Josh Broth, David A. Maltz, David B. Johnson, Yih-Chun Hu, and Jorjeta Jetcheva, "A performance comparison of multi-hop wireless ad hoc network routing protocols," Mobicom, 1998, pp. 85-97.

6. C.-L. Chang and R. C. T. Lee, Symbolic Logic and Mechanical Theorem Proving, Academic Press, New York, 1973.

7. B. N. Clark, C. J. Colbourn, and D. S. Johnson, "Unit disk graphs," Discrete Mathematics, vol. 86, pp. 165-177, 1990.

8. Bevan Das and Vaduvur Bharghavan, "Routing in ad-hoc networks using minimum connected dominating sets," IEEE International Conference on Communications, 1997, pp. 376-380.

9. J. Díaz, M. D. Penrose, J. Petit, and M. Serna, "Convergence theorems for some layout measures on random lattice and random geometric graphs," Combinatorics, Probability, and Computing, No. 6, pp. 489-511, 2000.

10. J. Díaz, M. D. Penrose, J. Petit, and M. Serna, "Approximating layout problems on random geometric graphs," Journal of Algorithms, vol. 39, pp. 78-116, 2001.

11. J. Díaz, J. Petit, and M. Serna, "Random geometric problems on $[0,1] 2$, "Lecture Notes in Computer Science, vol. 1518, Springer-Verlag, New York/ Berlin, 1998.

12. O. Dousse, P. Thiran, and M. Hasler, "Connectivity in ad-hoc and hybrid networks," Infocom, 2002.

13. P. Erdös and A. Rénye, "On Random Graphs I," Publ. Math. Debrecen, vol. 6, pp. 290297, 1959.

14. C. Fullmer and J. Garcia-Luna-Aceves, "Solutions to hidden terminal problems in wireless networks," ACM SIGCOMM, 1997, pp. 39-49.

15. E.N. Gilbert, "Random Graphs," Ann. Math. Stat., vol. 30, pp. 1141-1144, 1959.

16. M. C. Golumbic, Algorithmic Graph Theory and Perfect Graphs, Academic Press, New York, 1980.

17. A. Gräf, M. Stumpt, and G. Wei $\beta$ enfels, "On coloring unit disk graphs," Algorithmica, vol. 20, pp. 277-293, 1998. 
18. P. Gupta and P. R. Kumar, "Critical power for asymptotic connectivity in wireless networks," Stochastic Analysis, Control, Optimization and Applications, pp. 547-566, 1998.

19. P. Gupta and P. R. Kumar, "The capacity of wireless networks," IEEE Transactions on Information Theory, vol. 46, no. 2, pp. 388-404, 2000.

20. Peter Hall, Introduction to the Theory of Coverage Process, John Wiley and Sons, New York, 1988.

21. Paul G. Hoel, Sidney C. Port, and Charles J. Stone, Introduction to Probability Theory, Houghton Mifflin Company, Boston, Mass., 1971.

22. T. Hou and V. Li, "Transmission range control in multihop packet radio networks, "IEEE Transaction on Communications, vol. 34, pp. 38-44, 1986.

23. C.-F. Hsin and M. Liu, "Network coverage using low duty-cycled sensors: Random and coordinated sleep algorithm," International Symposium on Information Processing in Sensor Networks, 2004.

24. S. Khurana, A. Kahol, S. K. S. Gupta, and P. K. Srimani, "Performance evaluation of distributed co-ordination function for IEEE 802.11 wireless LAN protocol in presence of mobile and hidden terminals," International Symposium on Modeling, Analysis and Simulation of Computer and Telecommunication Systems, 1999, pp. 40-47.

25. S. Khurana, A. Kahol, and A. Jayasumana, "Effect of hidden terminals on the performance of the IEEE 802.11 MAC protocol," Proceedings of Local Computer Networks Conference, 1998.

26. L. Kleinrock and J. Silvester, "Optimum transmission radii for packet radio networks or why six is a magic number," Proc. IEEE National Telecom. Conf., 1978, pp. 4.3.1-4.3.5.

27. S.-J. Lee and M. Gerla, "AODV-BR: Backup routing in Ad hoc Networks," IEEE Wireless Communications and Networking Conference, 2000, vol. 3, pp. 1311-1316.

28. Edgar M. Palmer, Graphical Evolution: An Introduction to the Theory of Random Graphs, New York: John Wiley and Sons, 1985.

29. Mathew D. Penrose, Random Geometric Graphs, Oxford University Press, 2003.

30. M. D. Penrose, "A strong low for the longest edge of the minimal spanning tree," The Annals of Probability, vol. 27, no. 1, pp. 246-260, 1999.

31. M. D. Penrose, "The longest edge of the random minimal spanning tree," The Annals of Applied Probability, vol. 7, no. 2, pp. 340-361, 1997.

32. M. D. Penrose, "On $k$-connectivity for a geometric random graph," Random structures and Algorithms, vol. 15, no. 2, pp. 145-164, 1999.

33. T. K. Philips, S. S. Panwar, and A. N. Tantawi, "Connectivity properties of a packet radio network model," IEEE Transactions on Information Theory, pp. 1044-1047, 1989.

34. P. Piret, "On the connectivity of radio networks," IEEE Transactions on Information Theory, pp. 1490-1492, 1991.

35. G. J. Pottie and W. J. Kaiser, "Wireless integrated network sensors," Commun. ACM, vol. 43, no. 5, pp. 51-58, May 2000.

36. V. Ravelomanana, "Extremal Properties of three-dimensional sensor networks with applications," IEEE Transactions on Mobile Computing, vol. 3, no. 3, pp. 246-257, 2004.

37. F. S. Roberts, "Indifference graphs," in Proof Techniques in Graph Theory, F. Harary (editor), Academic Press, New York, pp. 139-146, 1969.

38. E.M. Royer and C-K Toh, "A Review of Current Routing Protocols for Ad Hoc Mobile Wireless Networks,“'IEEE Personal Communication, pp. 46-55, 1999.

39. Paolo Santi and Douglas M. Blough, "The critical transmitting range for connectivity in sparse wireless ad hoc networks," IEEE Transactions on Mobile Computing, vol. 2, no. 1, pp. 25-39, 2003.

40. Paolo Santi and Doulas M. Blough,"A probabilistic analysis for the radio range assignment problem in ad hoc networks," MobiHoc, 2001, pp. 212-220. 
41. D. Shukla, L. Chandran-Wadia, and S. Iyer, "Mitigating the exposed node problem in IEEE 802.11 ad hoc networks," International Conference on Computer Communications and Networks, 2003, pp. 157-162.

42. K. Sohrabi, J. Gao, V. Ailawadhi, and G. J. Pottie, "Protocols for self-organization of a wireless sensor network, "IEEE Personal Commun., vol. 7, no. 5, pp. 16-27, Oct. 2000.

43. J. Stewart, Calculus, 4th ed., Gary W. Ostedt, 1999.

44. H. Takagi and L. Kleinrock, "Optimal transmission ranges for randomly distributed packet radio terminals," IEEE Transaction on Communications, vol. 32, pp. 246-257, 1984.

45. F. Tobagi and L. Kleinrock, "Packet switching in radio channels, Part II-The hidden terminal problem in carrier sense multiple access and the busy tone solution," IEEE Trans. Commun., vol. COM-23, no. 12, pp. 1417-1433, 1975.

46. J. $\mathrm{Wu}$ and $\mathrm{H}$. Li, "Domination and its application in ad hoc wireless networks with unidirectional links," International Conference on Parallel Processing, 2000, pp. 189 197.

47. F. Xue and P. R. Kumar, "The number of neighbors needed for connectivity of wireless networks," Wireless Networks, vol. 10, pp. 169-181, 2004.

48. K. J. Yang, On the Number of Subgraphs of a Random Graph in [0, 1]d, Unpublished D.Phil. thesis, Department of Statistics and Actuarial Science, University of Iowa, 1995.

49. L.-H. Yen and C. W. Yu, "Link probability, network coverage, and related properties of wireless ad hoc networks," The 1st IEEE International Conference on Mobile Ad-hoc and Sensor Systems, 2004, pp. 525-527.

50. C. W. Yu, L.-H. Yen, and Yang-Min Cheng, "Computing subgraph probability of random geometric graphs with applications in wireless ad hoc networks," Tech. Rep., CHU-CSIETR-2004-005, Chung Hua University, R.O.C.

51. Chang $\mathrm{Wu} \mathrm{Yu}, \mathrm{Li}-\mathrm{H} s i n g$ Yen, Kun-Ming Yu, and Zhi Pin Lee, "An Ad Hoc Routing Protocol Providing Short Backup Routes," Eighth IEEE Internation Conference on Communication Systems, 2002, Singapore, pp.1052-1056.

52. Kun-Ming V. Yu, Shi-Feng Yand, and Chang Wu Yu, "An Ad Hoc Routing Protocol with Multiple Backup Routes," Proceedings of the IASTED International Conference Networks, Parallel and Distributed Processing, and Applications, 2002, pp. 75-80.

53. The Bluetooth Interest group," http://www.bluetooth.com." 